\title{
CHERNOFF'S THEOREM FOR BACKWARD PROPAGATORS AND APPLICATIONS TO DIFFUSIONS ON MANIFOLDS
}

\author{
EVELINA SHAMAROVA
}

Abstract. The classical Chernoff's theorem is a statement about discrete-time approximations of semigroups, where the approximations are consturcted as products of time-dependent contraction operators strongly differentiable at zero. We generalize the version of Chernoff's theorem for semigroups proved in [3] (see also [4] and [5]), and obtain a theorem about discrete-time approximations of backward propagators.

Mathematics subject classification (2010): 47D06, 47D07.

Keywords and phrases: Chernoff's theorem, backward propagator, diffusion on a manifold, generator.

\section{REFERENCES}

[1] A. Gulis Ashvili, J. A. CASteren, Non-autonomous Kato classes and Feynman-Kac propagators, World Scientific Publishing Co. Pte. Ltd., 2006.

[2] G. Nickel, R. Schnaubelt, An extension of Kato's stability condition for nonautonomous Cauchy problems, Taiwanese J. Math. 2, 4 (1998), 483-496.

[3] O. G. Smolyanov, H. v. Weizs ÄCKeR, O. Wittich, Brownian motion on a manifold as a limit of stepwise conditioned standard Brownian motions, Canadian Mathematical Society, Conference Proceedings, Vol. 29, 2000, pp. 589-602.

[4] O. G. Smolyanov, H. v. Weizs ÄCKer, O. Wittich, Chernoff's theorem and the construction of Semigroups, In Evolution Equations: Applications to Physics, Industry, Life sciences and Economics - EVEQ 2000, M. Ianelli, G. Lumer (eds.), pages 355-364. Birkhäuser, 2003.

[5] O. G. Smolyanov, H. v. Weizs Äcker, O. Wittich, Chernoff's Theorem and Discrete Time Approximations of Brownian Motion on Manifolds, Potential Analysis 26, 1 (2007), 1-29.

[6] O. TAKEYAMA, Asymptotic properties of asymptotically homogeneous diffusion processes on a compact manifold, J. Math. Soc. Japan 37, 4 (1985), 637-650.

[7] I. V. TelyatNikOV, Smolyanov-Weizsäcker surface measures generated by diffusions on the set of trajectories in Riemannian manifolds, Infinite Dimensional Analysis, Quantum Probability and Related Topics 11, 1 (2008), 21-31 